\title{
Quantum Signature in Anisotropic Singularities of Dihedral Energy in Hydrogen Bond Breaking of Water Dimer
}

\author{
$\operatorname{Md~} \operatorname{Ali}^{1}$ \\ ${ }^{1}$ Icahn School of Medicine at Mount Sinai
}

September 23, 2020

\begin{abstract}
Molecular simulations from small molecules to large bio-macromolecules and polymer systems are routinely used to simulate thermodynamics properties of interests by molecular mechanics-based potentials. In a recent paper, via three different semiempirical methods, we reported quantum singularities in dihedral energies as signature of chemical bond break-up process revealed under experimental X-ray as broken chemical moieties. In this present work, applying first principle methods of Hartree-Fock, Density Functional as well as MollerPlesset techniques we have reconfirmed the previous general predictions of singularities in in dihedral energies for the case of water dimer that connects two water monomers by weak hydrogen bond. Due to quantum nature of chemical bond breaking process leading to break-point conditions in otherwise connected molecular topology, the singularities in dihedral energies are also suggestive of large forces as onset in the bond-breaking process. We have presented the details of these novel interesting findings in this paper. These results of quantum singularities can have significant impacts to improve current force fields and can open up new areas we define as "Fracture Molecular Mechanics" or "Fracture Force Field" in overlap regions of molecular and quantum mechanics based approaches to explore and account for chemical bond-breaking mechanisms in molecular simulation techniques.
\end{abstract}

\section{Hosted file}

Water_Dimer_manuscript.pdf available at https://authorea.com/users/361388/articles/482786quantum-signature-in-anisotropic-singularities-of-dihedral-energy-in-hydrogen-bondbreaking-of-water-dimer 
Title: Quantum Signature in Anisotropic Singularities of Dihedral Energy in Hydrogen Bond Breaking of Water Dimer

1. Md Rejwan Ali,

Department of Medicine, Icahn School of

Medicine at Mount Sinai, New York, NY 10029, USA 
Title: Quantum Signature in Anisotropic Singularities of Dihedral Energy in Hydrogen Bond Breaking of Water Dimer

1. Md Rejwan Ali,
Department of Medicine, Icahn School of
Medicine at Mount Sinai, New York, NY 10029, USA* 